\title{
An Improved Current-Mode Squarer/Divider Circuit for Automotive Applications
}

\author{
Xin YIN $^{\dagger \text { a) }}$, Peter OSSIEUR ${ }^{\dagger}$, Tine De RIDDER ${ }^{\dagger}$, Nonmembers, Johan BAUWELINCK $^{\dagger}$, \\ Xing-Zhi QIU ${ }^{\dagger}$, Members, and Jan VANDEWEGE ${ }^{\dagger}$, Nonmember
}

SUMMARY A current-mode squarer/divider circuit with a novel translinear cell is presented for automotive applications. The proposed circuit technique increases the accuracy of the squarer/divider function with better input dynamic range and temperature insensitivity. Simulation results show that the variation of the output current is within $\pm 0.2 \%$ over the temperature range from $-40^{\circ} \mathrm{C}$ to $140^{\circ} \mathrm{C}$.

key words: current-mode circuits, translinear, squarer/divider circuit, BiCMOS

\section{Introduction}

A squarer/divider circuit is a common building block in analog signal-processing applications. For automotive applications, wide ambient temperature range (from $-40^{\circ} \mathrm{C}$ to $105^{\circ} \mathrm{C}$ ) and EMI suppression place strict requirements on circuit implementation. To overcome the hostile automotive environment, current-mode operation is normally used because of its out-performance (essentially insensitive to variations in bias level and temperature [1]) over counterparts that implemented in voltage-mode. Figure 1 shows the basic current-mode squarer/divider circuit. Assuming that the four transistors have the same emitter area, infinite current gain and infinite output resistance, the output current is given by reference [1]

$$
I_{\text {out }}=I_{x}^{2} / I_{u}
$$

However the finite current gain of bipolar transistors will introduce errors into this relationship. This is especially important here as transistor $Q_{3}$ is forced by emitter current $I_{u}$ while the other transistors are biased with collector currents. Due to the non-zero (temperature dependent) basecollector voltage and early effect of transistors $Q_{3}$ and $Q_{4}$, the ideal relationship will suffer from further errors. Furthermore for several applications, the output resistance of the normal squarer/divider circuit is not high enough.

\section{Proposed Squarer/Divider Circuit}

To force the collector current instead of the emitter current of $Q_{3}$, an opamp can be used as shown in Fig. 2(a). The opamp will bias $Q_{3}$ and eliminate its early effect by forcing

Manuscript received August 21, 2007.

Manuscript revised October 4, 2007.

${ }^{\dagger}$ The authors are with the Ghent University, INTEC/IMEC, St. Pietersnieuwstraat 41, Gent B-9000, Belgium.

a)E-mail: xin.yin@intec.ugent.be

DOI: $10.1093 /$ ietele/e91-c.2.232

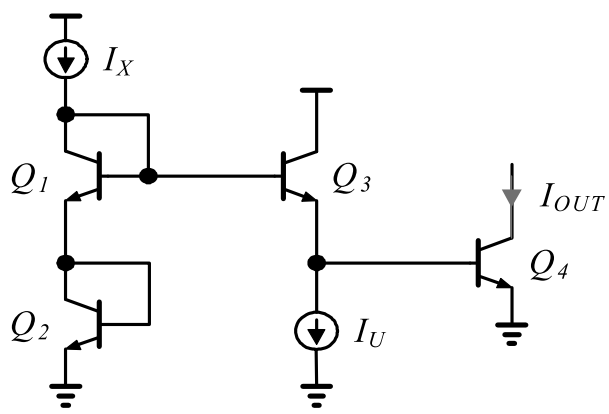

Fig. 1 Basic current-mode squarer/divider circuit.

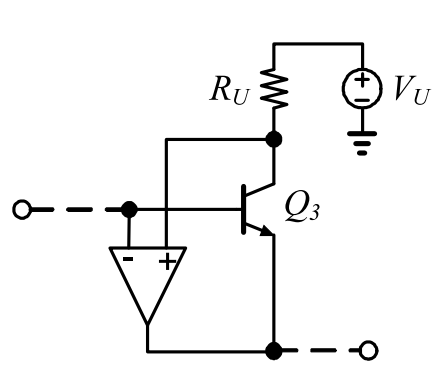

(a)

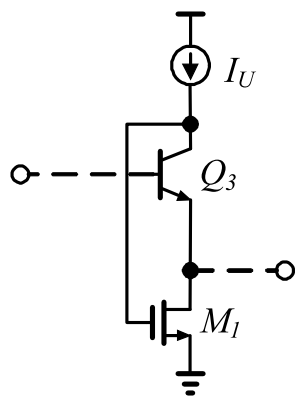

(b)
Fig. 2 (a) Opamp based scheme, (b) NMOS biased scheme, to force the collector current.

$V_{B C}$ to $0 \mathrm{~V}$. However it cannot be directly used in a currentmode circuit as its input signal is a voltage. Furthermore the common-base transistor $Q_{3}$ inserts additional gain into the feedback path and introduces instability [1]. An alternative way to force collector current is shown in Fig. 2(b) [2], in which the NMOS transistor $M_{1}$ is used to bias $Q_{3}$. It has as disadvantages that a BiCMOS process has to be used and that $Q_{3}$ will suffer from early effect as the bias voltage generated by $M_{1}$ will vary with NMOS corners and temperature.

We propose a novel translinear cell with a new bias scheme to force the collector current (Fig. 3). The bipolar transistor $Q_{5}$ and diode connected transistor $Q_{6}$ replace NMOS transistor $M_{1}$. As such, the base and collector voltage of $Q_{3}$ will roughly be equal to $2 V_{B E}$ and hence the error due to early effect is minimized. Current source $I_{b}$ is added to compensate for the error due to base current. 
The complete current-mode squarer/divider circuit is shown in Fig. 4. It consists of the squarer/divider core, a replica bias circuit and current mirrors. The squarer/divider core uses the proposed translinear cell to force the collector current instead of emitter current of $Q_{3}$. Cascode transistor $Q_{7}$ is used to increase the output resistance of the squarer/divider circuit. The base voltage of $Q_{7}$ is a replica of the base voltage of $Q_{3}$, which is generated by $Q_{8}$ in the replica bias circuit. Therefore the base and collector volt-

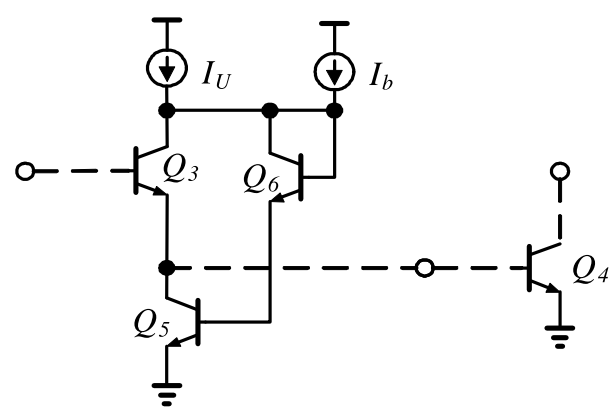

Fig. 3 Proposed translinear ell biased with collector current.

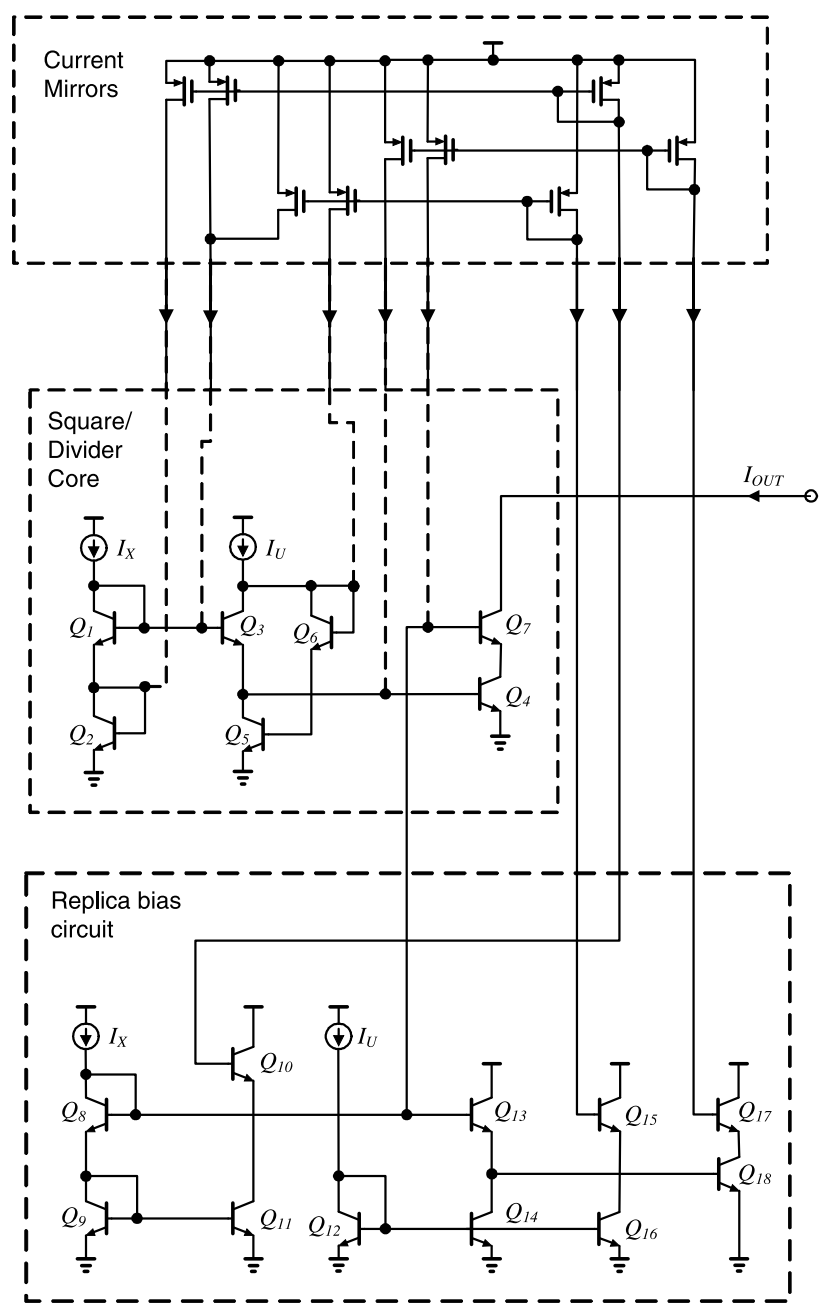

Fig. 4 Complete schematic of current-mode squarer/divider circuit. age of $Q_{4}$ will roughly be equal to one $V_{B E}$, i.e. $Q_{4}$ is also free of the early effect. Transistors $Q_{10}, Q_{15}$, and $Q_{17}$ in the replica bias circuit generate reference currents for base current compensation. The current mirrors then take these reference base currents and supply their copies back into appropriate base points.

\section{Simulations Results and Conclusion}

The relative errors between the expected current according to Eq. (1) and the squarer/divider output current are plotted versus input current $I_{x}$ for two cases of $I_{u}$ in Fig. 5 . Our proposed circuit outperforms the simple current-mode squarer/divider for all values of the input current, especially for extreme cases. Therefore it has much better input dynamic range. Also Fig. 6 shows proposed circuitry's temperature insensitivity. The variation of the output current is within $\pm 0.2 \%$ over the junction temperature range from $-40^{\circ} \mathrm{C}$ to $140^{\circ} \mathrm{C}$ (for ambient temperature range from $-40^{\circ} \mathrm{C}$ to $105^{\circ} \mathrm{C}$ ).

The simulation results demonstrate the improvement

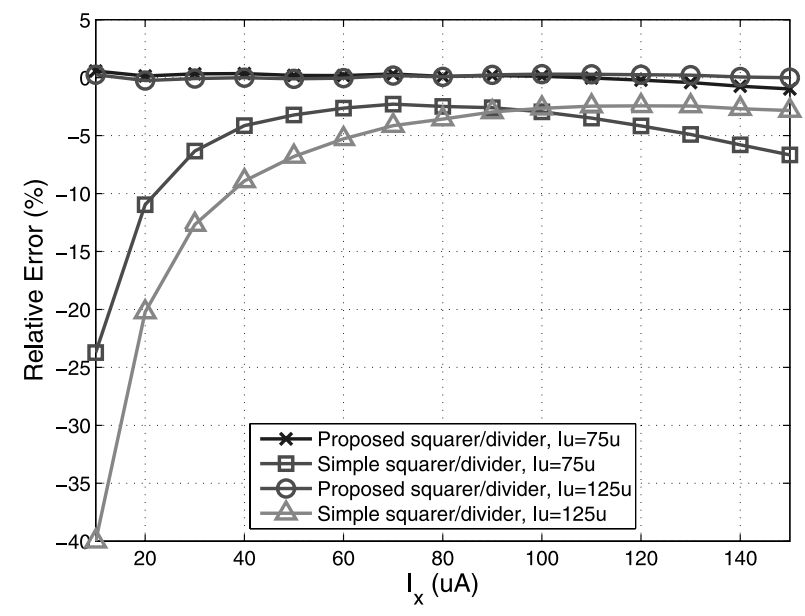

Fig.5 Relative error versus different input currents $I_{x}$.



Fig. 6 Variation of output current over the temperature range, $I_{x}=100 \mu$, $I_{u}=100 \mu$. 
in temperature insensitivity and dynamic range of our proposed circuit, though the price of increased area and current consumption is paid. Power consumption can be further reduced by scaling down the duplicated currents in replica bias circuit. And increase in area is not essentially a problem because the area of squarer/divider circuit is relatively small compared to automotive transmitter/receiver itself (e.g. about $1.2 \%$ of total area of our transmitter chip, implemented in XFAB 0.6 $\mu \mathrm{m}$ BiCMOS technology).

\section{References}

[1] B. Gilbert, "Current-mode circuits from a translinear viewpoint: A tutorial," in Analogue IC Design: The Current-Mode Approach, eds. C. Toumazou, F.J. Lidgey, and D.G. Haigh, ch. 2, pp.11-91, IEE Circuits and Systems Series 2, IEE London, 1990.

[2] M. Punzenberger and C.C. Enz, "A 1.2-V low-power BiCMOS class AB log-domain filter," IEEE J. Solid-State Circuits, vol.32, pp.19681978, Dec. 1997. 